Iman Attia, Ozan Zakariya Keskinkılıç, Büşra Okcu Muslimischsein im Sicherheitsdiskurs 
Iman Attia (Dr. phil.) ist Professorin an der Alice Salomon Hochschule Berlin. Sie lehrt und forscht zur Relationalität von Rassismen und ihren Intersektionen mit anderen gesellschaftlichen Machtverhältnissen. Ihr Schwerpunkt liegt neben Erinnerungsarbeit und globalhistorischen Narrativen vor allem auf antimuslimischem Rassismus.

Ozan Zakariya Keskinkılıç ist Politikwissenschaftler und Rassismusforscher. Seine Forschungsschwerpunkte sind u.a. (antimuslimischer) Rassismus, Antisemitismus, Orientalismus und Empowerment.

Büşra Okcu ist Praxisforscherin der Sozialen Arbeit und Pädagogik. Ihre Schwerpunkte liegen auf rekonstruktiven Methoden aus rassismuskritischer Perspektive, antimuslimischem Rassismus und Beratungsprozessen in der muslimischen Sozialen Arbeit. 
Iman Attia, Ozan Zakariya Keskinkılıç, Büşra Okcu

\section{Muslimischsein im Sicherheitsdiskurs}

Eine rekonstruktive Studie über den Umgang mit dem Bedrohungsszenario

Unter Mitarbeit von Ouassima Laabich-Mansour

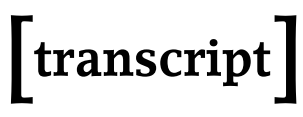


Die vorliegende Publikation wurde aus Mitteln des Bundesministeriums für Bildung und Forschung als Teilprojekt »Gouvernementalität« des Verbundprojekts »Das Bedrohungsszenario des sislamistischen Terrorismus aus den Perspektiven von Politik, Medien und muslimischen Communities - eine empirische Studie« unter dem Kennzeichen o1UG1830BX gefördert.

GEFŌRDERT VOM

拸1 $\begin{aligned} & \text { Bundesministerium } \\ & \text { für Bildung } \\ & \text { und Forschung }\end{aligned}$

\section{Bibliografische Information der Deutschen Nationalbibliothek}

Die Deutsche Nationalbibliothek verzeichnet diese Publikation in der Deutschen Nationalbibliografie; detaillierte bibliografische Daten sind im Internet über http://dnb.d-nb.de abrufbar.

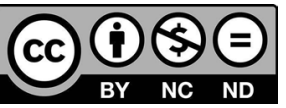

Dieses Werk ist lizenziert unter der Creative Commons Attribution-NonCommercial-NoDerivs 4.0 Lizenz (BY-NC-ND). Diese Lizenz erlaubt die private Nutzung, gestattet aber keine Bearbeitung und keine kommerzielle Nutzung. Weitere Informationen finden Sie unter https://creativecommons.org/licenses/by-nc-nd/4.o/deed.de

Um Genehmigungen für Adaptionen, Übersetzungen, Derivate oder Wiederverwendung zu kommerziellen Zwecken einzuholen, wenden Sie sich bitte an rights@transcript-publishing.com

Die Bedingungen der Creative-Commons-Lizenz gelten nur für Originalmaterial. Die Wiederverwendung von Material aus anderen Quellen (gekennzeichnet mit Quellenangabe) wie z.B. Schaubilder, Abbildungen, Fotos und Textauszüge erfordert ggf. weitere Nutzungsgenehmigungen durch den jeweiligen Rechteinhaber.

\section{() 2021 transcript Verlag, Bielefeld}

Umschlaggestaltung: Kordula Röckenhaus, Bielefeld

Umschlagcredit: »WE MUST NOT BE EXTREME« 2017, Artwork by: Faisal Hussain, www.suspectobjects.com, Photograph: David Rowan

Lektorat: Julia Jürgens, Berlin

Korrektorat: Luisa Bott, Bielefeld

Druck: Majuskel Medienproduktion $\mathrm{GmbH}$, Wetzlar

Print-ISBN 978-3-8376-5711-1

PDF-ISBN 978-3-8394-5711-5

https://doi.org/10.14361/9783839457115

Gedruckt auf alterungsbeständigem Papier mit chlorfrei gebleichtem Zellstoff. Besuchen Sie uns im Internet: https://www.transcript-verlag.de Unsere aktuelle Vorschau finden Sie unter www.transcript-verlag.de/vorschau-download 